\title{
Impactos da busca de informações não relevantes na produtividade de profissionais de Tecnologia da Informação
}

\author{
Maria Albeti Vieira Vitoriano \\ Doutora; Universidade Federal de Brasília, Brasília, DF, Brasil; \\ albeti@gmail.com \\ Kelley Cristine Gonçalves Dias Gasque \\ Doutora; Universidade Federal de Brasília, Brasília, DF, Brasil; \\ kelleycristinegasque@hotmail.com
}

\begin{abstract}
Resumo: Este artigo é resultante de uma pesquisa de doutorado realizada com profissionais da área de infraestrutura de Tecnologia da Informação que trabalham no Distrito Federal. Utiliza-se metodologia de natureza quantiqualitativa, com aplicação de questionário e realização de entrevistas individuais de natureza semiestruturada. Os resultados mostram que esses profissionais estão conectados durante a maior parte do tempo e, em geral, acessam informações sem relevância para o trabalho imediato, fenômeno denominado cyberloafing. Os principais motivos para o acesso são: atualização sobre os últimos acontecimentos, redução do nível de estresse e aumento do nível de conhecimento técnico. Reconhece-se que o acesso à internet desvia a atenção, mesmo assim muitos profissionais criticaram as organizações em que trabalham devido à prática de bloqueio de sites e de conteúdos. Os principais impactos no trabalho são o pouco aproveitamento do tempo para as tarefas na empresa e desvio da atenção. As organizações precisam se adaptar a essa nova realidade e encontrar soluções de gerenciamento e controle ao invés do uso exclusivo de ferramentas de bloqueio do acesso a certos tipos de sites e aplicativos.
\end{abstract}

Palavras-chave: Busca de informação. Cyberloafing. Informação não relevante. Informação relevante. Sobrecarga de informação.

\section{Introdução}

O uso da rede mundial de computadores tornou-se essencial, nos últimos anos, para o trabalho, para a escola e para a vida social (CARR, 2011). De acordo com Gonçalves (2017) e Knight (2014), a internet permite a disponibilização de inúmeros serviços e aplicativos para a população em geral, tanto pela iniciativa privada como pelos órgãos governamentais, em um movimento que se reforça mutuamente. No entanto, muitas informações acessadas pelos profissionais no 
ambiente de trabalho não são relevantes, o que pode causar prejuízo no desempenho das atividades (CARR, 2011).

Nesse contexto, entende-se informação relevante como aquela que tem utilidade imediata para a realização das atividades; por sua vez, informação não relevante é aquela sem importância para as atividades do indivíduo. Entretanto, Barry (1994) afirma que essa informação pode ter sido relevante em algum outro momento ou vir a ser no futuro, tendo em vista a natureza dinâmica das necessidades de informação do usuário.

$\mathrm{O}$ artigo apresenta os resultados de uma pesquisa de doutorado sobre os impactos da busca de informações não relevantes na produtividade de profissionais de Tecnologia da Informação (TI). O objetivo da pesquisa foi verificar o impacto da busca de informações não relevantes por meio da internet durante o horário de trabalho. Definiu-se, como universo de estudo, os profissionais que trabalham na área de infraestrutura de TI em organizações sediadas no Distrito Federal (DF).

A revisão de literatura abrangeu os temas: relevância da informação; busca da informação; uso da internet no trabalho e profissional de TI. Desses tópicos, selecionou-se o referencial teórico, que deu suporte às etapas da pesquisa. A pesquisa é exploratória, de natureza quanti-qualitativa. Os dados foram obtidos por meio de questionários e entrevistas semiestruturadas.

\section{Referencial teórico}

A informação é um fator estratégico para as organizações, usada para criar significado, construir conhecimento e tomar decisões, como também auxiliar na melhoria dos processos, produtos e serviços (TARAPANOFF, 2006). Castells (1999) defende que, na economia da informação, a produtividade e a competitividade organizacional dependem, primordialmente, da capacidade de gerar, processar e aplicar a informação de forma eficiente. A seguir, são apresentados os conceitos-chave da pesquisa. 


\subsection{Informação não relevante}

De acordo com os autores Schamber, Eisenberg e Nilan (1990) e Saracevic (2017), a noção e o julgamento do ser humano sobre a relevância envolvem a percepção do usuário e a situação em determinado momento. Portanto, a eficiência do indivíduo depende do que ele faz com a quantidade de informações a que tem acesso, o que é ignorado e o que é aceito, como armazena e como utiliza esse acervo para ações futuras em busca de conhecimento e sabedoria. De forma sintética, McGarry (1999) explica que a relevância de algo se relaciona ao discernimento e à rejeição do que não é relevante.

Em Shanteau (1992), informação não relevante é aquela que não tem relação, de forma direta, com determinado contexto e, portanto, não tem utilidade nessa situação. No entanto, essa mesma informação poderá ser útil, em outro momento, para o mesmo indivíduo. O grande desafio é saber distinguir entre o que é relevante numa determinada situação e o que não tem relevância,

Caso contrário, a informação sem relevância pode transformar-se em ruído, um obstáculo que deve ser erradicado, e tornar-se desinformação (SANTOS, 2005). Além disso, na visão de Hjelseth, (2010), informações não relevantes, em especial as detalhadas e extensas, podem conduzir o indivíduo a resultados falsos.

Para Lazarte (2000), a atenção ao que é fundamental auxilia na seleção das informações relevantes para cada contexto. Ele argumenta que o grande desafio do ser humano é aprender a ignorar o que não é relevante. Saber diferenciar as informações relevantes das não relevantes é crucial para o sucesso na resolução de problemas (COOK, 2006). Isso porque, conforme Pijpers (2010), a informação, ao invés de auxiliar o indivíduo a melhorar o trabalho, cria ameaças sobre o controle das tarefas a serem realizadas, podendo causar erros, inconsistências e gerar outras informações sem valor. 


\subsection{Busca de informação}

A busca de informações envolve, em linhas gerais, a procura, a extração e o uso de conteúdo para um propósito específico (WILSON, 1997; WILSON, 2000). Wilson (2000) argumenta que, nesse processo, são adotadas estratégias e critérios para decisão de qual material deve ser consultado e o julgamento da relevância das informações recuperadas. Gasque (2012), por sua vez, explica que buscar e usar a informação de forma eficaz e eficiente são competências cruciais, que podem ser desenvolvidas por meio do letramento informacional. A autora argumenta que a busca da informação relaciona-se ao modo como as pessoas procuram-na para suprir uma necessidade e requer envolvimento ativo ou passivo na sua busca, "o planejamento das atividades de busca, as estratégias e a motivação para atingir objetivos; a monitoração de estratégias; o conhecimento e a definição de canais ou fontes de informação potenciais; as competências para usar tecnologias da informação e a avaliação do processo" (GASQUE, 2012, p. 85)

Conforme Spink e Cole (2006), a ideia da busca de informação para solucionar problemas tem sido a abordagem dominante na Ciência da Informação (CI), mas existem outras abordagens de caráter interdisciplinar. Bates (1989) afirma que, nas pesquisas relacionadas à vida real, os usuários podem iniciar uma busca de informação, a partir de um tópico amplo ou com uma referência relevante e, em seguida, movimentar-se através de diversas fontes. A própria consulta e os termos de pesquisa utilizados podem ser continuamente modificados, em parte ou na totalidade.

Choo, Detlor e Turnbull (2000) identificam quatro modalidades de pesquisa na Internet: visualização direcionada, visualização condicionada, pesquisa formal e pesquisa informal. Bates (2002) e Savolainen (2016) fazem a integração entre o comportamento de busca e o comportamento de pesquisa de informação, com referências aos modos de pesquisa propostos por Wilson (1997) e Wilson (1999): atenção passiva, busca passiva, busca ativa e pesquisa contínua. 


\subsection{Uso da internet no ambiente de trabalho}

A introdução da internet nas organizações serve para fortalecer e catalisar processos de produção (LARA, 2006). Os dispositivos pessoais de internet móvel, como telefones celulares e tablets, expandem as possibilidades de conexão à internet a qualquer momento e em qualquer lugar (SAWITRI, 2012; JAMALUDDIN et al.,2015).

$\mathrm{Na}$ perspectiva desses autores, os empregados podem desviar parte do horário de trabalho para acesso à internet e, em consequência, causar prejuízo na realização das atividades. Esse comportamento, portanto, tem potencial para redução da produtividade dos profisssionais.

Alguns autores consideram o uso da internet no trabalho, para fins pessoais, comportamento censurável, que deve ser monitorado e controlado pelas organizações através de políticas de acesso à internet (BLAU; YANG; WARD-COOK, 2006). Outros autores, como Lim e Chen (2009), entendem que esse comportamento, em algumas situações, pode ser considerado como positivo, tendo em vista que pode reduzir o estresse e agregar diversidade às rotinas diárias.

De um modo geral, as organizações utilizam políticas para regulamentar o uso da internet e mecanismos técnicos para monitorar e controlar o acesso à internet pelos empregados (GLASSMAN; PROSCH; SHAO, 2015; KHANSA et al., 2017). No entanto, apesar da adoção e implantação de políticas e de mecanismos técnicos, Glassman, Prosch e Shao (2015) afirmam que o uso da internet para acessar informações não relevantes persiste nas organizações.

Blau, Yang e Ward-Cook (2006) entendem que o comportamento de uso da internet durante o trabalho, cyberloafing, pode estar fortemente relacionado com a percepção de justiça no tratamento interpessoal. Portanto, as organizações precisam repensar as estratégias, de forma a reduzir os efeitos desses dispositivos sobre o desempenho dos funcionários (AKU, 2017; GONÇALVES, 2017), principalmente considerando o potencial da web 2.0. 


\subsection{Profissional de tecnologia da informação}

A TI abrange a convergência das indústrias de computação, mídia e telecomunicações no contexto mais amplo relacionado à revolução gerada pelo computador (HAIGH, 2011). O profissional de TI é o indivíduo que atua nessa área, de forma remunerada, em diversas atividades, como análise de sistemas, suporte técnico, suporte operacional, programação de computador, implantação de sistemas, testes de sistemas, levantamento de requisitos, bem como gerência de projetos, de equipes e de recursos (MOURA JUNIOR ; HELAL , 2013).

\section{Metodologia}

A pesquisa estudou como os profissionais que trabalham em empresas localizadas no DF, na área de infraestrutura de TI, usam a internet no ambiente de trabalho. Os profissionais trabalhavam em serviços relacionados à infraestrutura, operações, arquitetura de centro de processamento de dados (data centers) e segurança da informação.

O estudo tem abordagem quanti-qualitativa e natureza exploratória. No último caso, conforme Braga (2007), não existem hipóteses a serem comprovadas, mas a busca de dados, informações, padrões, ideias ou suposições a respeito de um tema. Dividiu-se a pesquisa em duas fases: na primeira foi realizado levantamento exploratório, com utilização de questionário distribuído pelo Facebook, e-mail e WhatsApp; as perguntas iniciais do questionário identificaram o perfil dos respondentes, as demais identificaram a opinião dos profissionais sobre o acesso a informações não relevantes no ambiente de trabalho por meio da internet. Nas perguntas que buscavam conhecer a opinião dos profissionais, foram utilizadas as seguintes opções: "sempre", "frequentemente", "indeciso (a)", "raramente" e "nunca".

As palavras "sempre" e "nunca" foram colocadas nos extremos da escala. De acordo com Houaiss e Vilar (2009, p. 1727), "sempre" significa "na totalidade do tempo" e "nunca", "em nenhum tempo; jamais" (HOUAISS; VILAR, 2009, p. 1368). Para mensurar posições moderadas em relação aos extremos, foram usadas as palavras "frequentemente" e "raramente". 
$\mathrm{Na}$ segunda fase, foram realizadas entrevistas semiestruturadas, com uso de amostra intencional. De acordo com Creswell (2014), esse tipo de amostra caracteriza-se pela escolha de participantes com experiência no fenômeno que é estudado.

\section{Resultados}

Neste item, apresentam-se os resultados obtidos pelos dois instrumentos utilizados na pesquisa: questionário e entrevista semiestruturada. A coleta de dados por meio dos questionários foi realizada entre o período de 18 de julho 2017 a 13 de outubro de 2017. Foram recebidos 256 questionários preenchidos, dos quais foram selecionados 142 respondentes, que atenderam aos critérios: trabalhar no DF e na área de infraestrutura de TI.

Nem todas as respostas analisadas representam a opinião desse número de profissionais. Alguns não responderam algumas perguntas e/ou declararam-se "indeciso (a)". Por essa razão, considerou-se somente as respostas "sempre" e "frequentemente", englobadas como "frequente"; "raramente" e "nunca" foram unificadas como "raro/nunca".

Nas entrevistas, houve a participação de 28 profissionais que trabalhavam, na época da pesquisa, em três organizações, duas públicas e uma privada. As entrevistas foram realizadas entre 31 de agosto de 2017 e 13 de outubro de 2017. Após coleta dos dados, os questionários foram analisados por meio de instrumentos estatísticos, e as entrevistas, por meio de análise de conteúdo.

\subsection{Perfil demográfico}

Em relação aos questionários, dos 142 respondentes, 67,6\% (n=96) trabalhavam na administração pública; 58,5\% ( $\mathrm{n}=83)$, exerciam função técnica; 81,7\% ( $\mathrm{n}=116)$ pertenciam ao gênero masculino; 47,9\% (68) estavam na faixa etária entre 31 e 40 anos; e 54,9\% (78) tinham especialização. Nas entrevistas, manteve-se o mesmo perfil, com exceção do tipo de organização em que trabalhavam. Dos 28 entrevistados, 18 trabalhavam em uma empresa privada. 
Maria Albeti Vieira Vitoriano e Kelley Cristine Gonçalves Dias Gasque

A maioria dos profissionais que participaram da pesquisa faz parte da geração milênio ou nativos digitais, que nasceram a partir de década de 1980 (MILLION et al., 2013). De acordo com Lipkin e Perrymore (2010), os nativos digitais também podem ser chamados de geração Y, geração da internet ou igeração.

\subsection{Busca de informações não relevantes}

Os resultados mostram que, das 136 respostas válidas, 41,2\% (n=56) admitiram acessar informações não relevantes no horário de trabalho. Nas entrevistas, verificou-se que esse acesso ocorreu, principalmente, pelo telefone celular.

O aplicativo mais acessado foi o WhatsApp, seguido pelo Facebook e pelo Instagram. O WhatsApp foi utilizado para troca de mensagens com família e amigos, mas também para uso profissional. Nas três organizações, o aplicativo foi usado intensamente como ferramenta de comunicação no trabalho.

Nas organizações onde esses profissionais trabalhavam, havia bloqueio para acesso a alguns sites e aplicativos por meio da rede corporativa. Houve unanimidade sobre essa medida em relação aos sites de pornografia e outros conteúdos perigosos. Entretanto, a maioria admitiu que as organizações precisam rever esse tipo de comportamento, tendo em vista que o celular se tornou ferramenta de uso geral e pode acessar todo tipo de site e aplicativo dentro da organização.

Perguntados sobre a influência das informações não relevantes na realização das atividades, de 121 respostas válidas, 22,3\% (n=27) admitiram que havia interferência. Como visto anteriormente, 41,2\% ( $\mathrm{n}=56)$ afirmaram acessar esse tipo de informação. As buscas ocorriam sem objetivos definidos, tendo em vista que 77,7\% ( $\mathrm{n}=94)$ dos entrevistados, por exclusão, admitiram que essas informações não têm influência sobre as atividades em execução. Nessa questão, $18,6 \%(\mathrm{n}=19)$ declararam-se indecisos (as).

Com o objetivo de identificar as informações mais acessadas no horário de trabalho, foram apresentadas cinco categorias de informação: "lazer e entretenimento"; "oportunidades de trabalho"; "política e economia"; "novidades da área de TI"; e "conteúdo das redes sociais". As respostas obtidas trouxeram os seguintes resultados, em relação a essas categorias: "novidades da área de TI", 81,5\% (n=110 de 135); "política e economia", 44,7\% ( $\mathrm{n}=59$ de 132); "lazer e 
Maria Albeti Vieira Vitoriano e Kelley Cristine Gonçalves Dias Gasque

entretenimento", $24,8 \%(\mathrm{n}=32$ de 129); "conteúdo das redes sociais", 17,8\% (n=24 de 135); e "oportunidades de trabalho", $13,4 \%$ (n=18 de 134).

Em relação à relevância dessas informações para as atividades profissionais, foram obtidos os seguintes resultados: "lazer e entretenimento", 6,3\% ( $\mathrm{n}=8$ de 126); “conteúdo das redes sociais", 11,3\% (n=14 de 124); "oportunidades de trabalho", 14,2\% ( $\mathrm{n}=18$ de 127); "política e economia", 36,6\% ( $\mathrm{n}=45$ de 123); e "novidades da área de TI”, 91,2\% (n=125 de 137). Observou-se que as informações não relevantes relativas a "novidades da área de TI" aparecem como as mais acessadas e também como as mais relevantes para as atividades profissionais.

Dessa forma, pode-se inferir que, do ponto de vista dos respondentes, o acesso a esse tipo de informação não gera prejuízos para as atividades profissionais, provavelmente porque poderão ser relevantes para atividades futuras. De acordo com Saracevic (2017), o julgamento da relevância da informação não é objetivo nem estático, varia de acordo com o ambiente, o contexto e as circunstâncias. Portanto, deve-se considerar a relevância das informações a partir do ponto de vista dos profissionais de TI. De qualquer forma, deve-se entender que a busca dessas informações pode causar desvio de tempo e de foco das atividades em desenvolvimento, que são prioritárias para a organização. Sobre isso, Goleman (2014) enfatiza que a capacidade de atenção se relaciona ao nível de competência realizada em cada tarefa. Ele explica que a tecnologia captura a atenção das pessoas e interrompe as conexões cerebrais. Em síntese, o bom desempenho depende do foco do indivíduo.

$\mathrm{Na}$ tabela 1, apresentam-se as informações mais acessadas pelos respondentes em relação à relevância. Observou-se que as informações sobre "novidades da área de TI" e sobre "política e economia" foram as mais acessadas e também as consideradas mais relevantes.

Tabela 1 - Informações acessadas versus julgamento da relevância

\begin{tabular}{l|l|l|l|l}
\hline \multicolumn{1}{c|}{ Categorias } & Acesso $(\boldsymbol{\%})$ & Respostas (n) & Relevância (\%) & Respostas (n) \\
\hline Novidades da área de TI & 81,5 & 110 & 91,2 & 125 \\
\hline Política e economia & 44,7 & 59 & 36,6 & 45 \\
\hline Lazer e entretenimento & 24,8 & 32 & 6,3 & 8 \\
\hline Conteúdo das redes sociais & 17,8 & 24 & 11,3 & 14 \\
\hline Oportunidades de trabalho & 13,4 & 18 & 14,2 & 18 \\
\hline
\end{tabular}

Fonte: Elaborada pelas autoras. 
Maria Albeti Vieira Vitoriano e Kelley Cristine Gonçalves Dias Gasque

Nas entrevistas, confirmou-se a existência de muitos acessos a informações sobre "novidades da área de TI". Entretanto, grande parte dessas informações não foi relevante para as atividades em exercício e causou desvio da atenção em relação ao trabalho realizado.

Para mensurar o acesso a informações não relevantes no ambiente de trabalho, foram oferecidas cinco opções: "auxílio na realização das tarefas"; "aumento do nível de conhecimento técnico"; "redução do nível de estresse"; "atualização sobre os últimos acontecimentos"; e "interação nas redes sociais". As respostas apontaram para os seguintes resultados: "atualização sobre os últimos acontecimentos", 72,5\% (n=95 de 142); "redução do nível do estresse", 66,9\% ( $\mathrm{n}=85$ de 127); "aumento do nível de conhecimento técnico", 60,3\% ( $\mathrm{n}=73$ de 121); "auxílio na realização das tarefas", 35,3\% ( $\mathrm{n}=42$ de 119); e “interação nas redes sociais", 15,4\% (n=20 de 130).

Na tabela 2, apresentam-se os resultados decorrentes dos acessos em ordem decrescente: "atualização sobre os últimos acontecimentos", "redução do nível de estresse" e "aumento do nível de conhecimento técnico" foram os principais motivos por que os profissionais buscaram informação não relevante no horário de trabalho.

Tabela 2 - Resultados obtidos com o acesso a informações não relevantes

\begin{tabular}{l|c|c}
\hline Motivos & Acesso (\%) & Respostas (n) \\
\hline Atualização sobre os últimos acontecimentos & 72,5 & 95 \\
\hline Redução do nível do estresse & 66,9 & 85 \\
\hline Aumento do nível de conhecimento técnico & 60,3 & 73 \\
\hline Auxílio na realização das tarefas & 35,3 & 42 \\
\hline Interação nas redes sociais & 15,4 & 20 \\
\hline
\end{tabular}

Fonte: Elaborada pelas autoras.

Pode-se relacionar a necessidade de "atualização sobre os últimos acontecimentos" com a busca de informações sobre "política e economia", que foi avaliada como a segunda categoria de informações mais acessada. Nas entrevistas, a maioria destacou que essas informações foram importantes para atualizá-los sobre o que ocorre no país e no mundo.

Para os entrevistados, a necessidade de acesso a esse tipo de informação deve-se, em grande parte, ao momento conturbado que o país vive nos últimos três anos. Em geral, eles acessavam sites de notícias no início da manhã, antes 
Maria Albeti Vieira Vitoriano e Kelley Cristine Gonçalves Dias Gasque

do almoço e no final da tarde. Durante os demais horários, os acessos foram feitos sob demanda, na medida em que recebiam notificações - prioritariamente pelo celular. Eles admitiram que esse tipo de informação não foi relevante para a realização das atividades profissionais.

A "redução do nível de estresse" alcançada pelo acesso a informações não relevantes foi apontada por 66,9\% $(\mathrm{n}=85)$ de 127 respondentes. Os entrevistados também concordaram com o resultado, pois, segundo eles, além de ajudar na redução do estresse, o cyberloafing serve como distração, descanso da mente e diminuição da tensão no ambiente de trabalho.

Por outro lado, uma entrevistada destacou que sentia ansiedade e ficava estressada quando se deparava com muitas informações; assim, preferia ignorálas, mesmo correndo o risco de perder informações relevantes. Million et al. (2013) defendem que a diversidade de mecanismos para acessar informações potencializa situações de cansaço e estresse, mas, por outro lado, cria oportunidades para descoberta de informação por acaso.

Lim e Chen (2012) argumentam que, em algumas situações, o uso da internet para fins pessoais pode ser considerado positivo, tendo em vista que pode reduzir o estresse e agregar diversidade à rotina diária. Nesse sentido, pode ser considerado como um intervalo periódico para os profissionais recarregarem o nível de energia e aumentarem o desempenho no trabalho.

No que se refere ao acesso a informações para "aumento do nível de conhecimento técnico" e "auxílio na realização das tarefas", essas respostas podem estar relacionadas à categoria de informação "novidades da área de TI". No entanto, esses dois aspectos ficaram na terceira e na quarta posição, respectivamente. Enquanto isso, a categoria "novidades da área de TI" foi apontada como a mais acessada e também considerada relevante por um alto percentual de respondentes.

Portanto, percebe-se incoerência na relação entre essas duas variáveis. Assim sendo, os acessos a esse tipo de informação podem estar, em grande parte, aliados aos interesses pessoais, alheios às atividades inerentes à função e, portanto, gerando impactos para a execução das tarefas daquele momento.

Finalmente, solicitou-se a opinião dos respondentes sobre os resultados advindos do acesso a informações não relevantes para a "interação nas redes 
Maria Albeti Vieira Vitoriano e Kelley Cristine Gonçalves Dias Gasque

sociais". Do total de 130 respostas válidas, $15,4 \%(n=20)$ opinaram que, com frequência, as informações não relevantes trazem resultados nos relacionamentos nas redes sociais.

Nas entrevistas, houve referências a informações do Facebook e do Instagram para manutenção da rede de relacionamentos. Porém, o que mais ocorreu foi a troca de mensagens pelo WhatsApp para comunicação com a família e os amigos. De acordo com Lipkin e Perrymore (2010), para a geração Y, o contato com os amigos é tão essencial que não é possível para a organização tentar evitar que isso ocorra no horário de trabalho.

Os entrevistados, em geral, justificaram os acessos a informações não relevantes durante o horário de trabalho como uma forma de manter-se atualizado sobre novas tecnologias, desligar-se de rotinas e/ou problemas do trabalho, buscar conhecimento em geral, estar constantemente conectado e também pela curiosidade sobre o que ocorre fora do ambiente de trabalho. A necessidade de estar conectado mostra que os dispositivos móveis são poderosos e mudam não apenas o que as pessoas fazem, mas o que são. Turkle (2012) explica que, muitas vezes, as pessoas sentem-se confortáveis por estarem online e cuidadosamente mantidas à distância das outras pessoas. Ou seja, a conexão pode ser também um esconderijo para ficar a uma distância segura. Isso porque os relacionamentos humanos são tão ricos quanto confusos e exigentes, sendo que a tecnologia permite uma "fuga".

Em relação ao tempo de acesso à internet para busca de informações não relevantes, foram obtidas 140 respostas válidas. O maior percentual de respondentes, 34,3\% ( $\mathrm{n}=48)$ indicou que acessa a internet entre 15 e 30 minutos; 25,7\% ( $\mathrm{n}=36)$, menos de 15 min. Foram assinaladas, porém, algumas utilizações entre uma e duas horas e também acima de duas horas.

De acordo com informações coletadas nas entrevistas, 52 min é o tempo médio diário de uso da internet para acesso a informações não relevantes. Além desse tempo, todos reportaram constantes interrupções causadas pelo telefone celular, principalmente em função do WhatsApp.

Nas entrevistas, percebeu-se que os profissionais consideravam acesso à internet no horário de trabalho somente aquele feito pelo computador, usando a 
Maria Albeti Vieira Vitoriano e Kelley Cristine Gonçalves Dias Gasque

rede de dados da organização. Portanto, é possível que essa interpretação tenha influenciado, para menor, os tempos de acesso obtidos nos questionários.

\section{Conclusões e recomendações}

Os dados coletados por meios dos questionários e das entrevistas mostram que existe muito acesso a informações não relevantes durante o horário de trabalho. As informações mais acessadas são: "novidades da área de TI"; "política e economia"; e "lazer e entretenimento".

Dessas informações, "novidades da área de TI" e "política e economia" são consideradas, pela maioria dos respondentes, como relevantes. A categoria "lazer e entretenimento" foi considerada como a menos relevante dentre as cinco categorias avaliadas. Essa diferença importante entre o nível de acesso e o julgamento da relevância dessas informações aponta muito fortemente para desvio do tempo das funções laborais.

Em relação aos resultados obtidos pelo acesso a informações não relevantes, o maior percentual obtido relaciona-se com a "atualização sobre os últimos acontecimentos". Esse resultado é coerente com o percentual de utilização obtido pela categoria de informação "política e economia", que ficou em segundo lugar na indicação de informações mais acessadas.

O "aumento do nível de conhecimento técnico" e o "auxílio na realização das tarefas", resultados que deveriam ser relacionados ao acesso a "novidades da área de TI", aparecem na terceira e quarta posição, respectivamente. Essa categoria de informação obteve altos percentuais em relação ao nível de acesso e ao julgamento de relevância. A diferença nos percentuais entre essa categoria e aqueles resultados alcançados pode indicar que os profissionais acessam informações para atender, mais diretamente, a interesses fora do escopo das atividades correntes e, portanto, com prejuízo para o trabalho.

Quanto à "redução do nível de estresse", obteve-se alto percentual nos questionários e também nas entrevistas. $\mathrm{O}$ resultado pode estar relacionado com a categoria "lazer e entretenimento". No que se refere à "interação nas redes sociais", o percentual obtido nesse item foi baixo e está relacionado com a categoria "conteúdo das redes sociais". Os entrevistados destacaram a 
necessidade de se manterem atualizados em relação ao que ocorre nas redes sociais e à troca de mensagens pelo WhatsApp.

Outro aspecto a considerar, quando se analisa as consequências desse tipo de comportamento, é o fator tempo. Mais de um terço dos entrevistados admitiram utilizar-se da internet de 15 a 30 minutos para acessar informações não relevantes. No entanto, existe a possibilidade de que nesse tempo esteja computado somente os acessos feitos por microcomputador. A literatura aponta, porém, que os acessos por telefone celular são os mais comuns e frequentes, sendo que praticamente todos são direcionados à busca de informações não relevantes. De qualquer forma, chamou a atenção o percentual de $14,3 \%$ de profissionais que admitiram usar a internet entre uma e duas horas para acessar informações não relevantes.

A pesquisa enfrentou algumas limitações que dificultaram a obtenção de resultados mais conclusivos. A primeira dessas restrições, comum em trabalhos desta natureza, foi a dificuldade em conseguir indivíduos que se disponham a participar de uma pesquisa acadêmica; outra restrição foi o fato da pesquisa ter relação com o comportamento de profissionais no ambiente de trabalho, o que excluiu vinculação com qualquer organização. Esses aspectos dificultaram a coleta de dados no questionário e, principalmente, nas entrevistas.

Outra limitação foi a dificuldade de mapear a quantidade de profissionais de TI que trabalham no DF, tendo em vista não ser uma profissão regulamentada e não ter um órgão representativo da categoria. Essas dificuldades foram superadas, na medida do possível, por meio da rede de relacionamento de uma das pesquisadoras.

Os dados coletados, nos questionários e nas entrevistas, apontam para a existência de impactos da busca de informações não relevantes na produtividade de profissionais de tecnologia da informação. Tal tema é atual e crítico para as organizações, portanto merece que outros estudos sejam desenvolvidos em relação às diversas profissões e organizações, principalmente em relação ao uso do telefone celular.

Muitas organizações limitam e controlam o acesso a alguns tipos de sites, correio eletrônico e redes sociais por meio de tecnologias que bloqueiam o acesso via rede corporativa. No entanto, as ações não são efetivas, na medida em 
que todos os acessos podem ser efetuados pelos telefones celulares, de propriedade dos indivíduos e sobre os quais as organizações não têm controle. Em consequência, perde-se o foco e desperdiça-se o tempo.

Portanto, as organizações precisam repensar novas formas de lidar com essa realidade, que vão além do uso de ferramental tecnológico e/ou eventuais medidas de caráter disciplinar. Novos modelos de gestão precisam ser aplicados, diferentes do modelo tradicional de comando e controle. As estruturas e processos organizacionais da sociedade, que, cada vez mais, organizam-se em redes de base tecnológica, precisam acompanhar esse direcionamento, no qual se inclui a convivência com a internet e tudo que ela representa.

Alguns sites e aplicativos devem ter o acesso bloqueado, tais como sites de pornografia, jogos eletrônicos e alguns outros que possam causar problemas de segurança para os serviços e produtos das organizações. No entanto, o acesso a outros tipos de sites e de aplicativos poderiam ser melhor gerenciado, $a$ posteriori, com o uso de relatórios gerenciais e estratégia de acompanhamento da produtividade dos profissionais.

Cabe, portanto, que o tema seja investigado com mais profundidade e maior abrangência, tendo em vista que a tecnologia da internet, a enorme quantidade de informações disponível e os diversos meios de acesso - notadamente, o celular são fenômenos irreversíveis. Porém, são recursos que podem ser muito bem utilizados no ambiente de trabalho desde que devidamente gerenciados.

\section{Referências}

AKU, Anizizo. Role of middle managers in mitigating employee cyberloafing in the workplace. 2017. Dissertation (Doctor of Philosophy Management) - College of Management and Technology, Walden University, Minnesota, 2017.

BARRY, Carol L. User-defined relevance criteria: an exploratory study. Journal of the American Society for Information Science, Baton Rouge, v. 45, n. 3, abr. 1994.

BATES, Marcia. The design of browsing and berrypicking techniques for the online search interface. Online Review, Los Angeles, v. 13, n. 5, p. 407-424. 1989. 
BATES, Marcia. Toward an integrated model of information seeking and searching. New Review of Information Behaviour Research, Los Angeles, v. 3, p. 1-15, 2002.

BLAU, Gary; YANG, Yang; WARD-COOK, Kory. Testing a measure of cyberloafing. Journal of Allied Health, Philadelphia, v. 35, n. 1, fev., p. 9-17, 2006.

BRAGA, Kátia S. Aspectos relevantes para a seleção de metodologia adequada à pesquisa social em Ciência da Informação. In: MUELLER, Suzana P. M. (Org.). Métodos para pesquisa em Ciência da Informação. Brasília: Thesaurus, 2007.

CARR, Nicholas. A geração superficial: o que a internet está fazendo com os nossos cérebros. Rio de Janeiro: Agir, 2011.

CASTELLS, Manuel. A sociedade em rede - A era da informação: economia, sociedade e cultura. São Paulo: Paz e Terra, 1999.

CHOO, Chun W.; DETLOR, Brian; TURNBULL, Don. Information seeking on the web: an integrated model of browsing and searching. First Monday, Chicago, v. 5, n. 2, fev., 2000.

COOK, Joan L. College students and algebra story problems: strategies for identifying relevant information. Reading Psychology, Philadelphia, v. 27, n. 23, p. 95-125, 2006.

CRESWELL, John W. Investigação qualitativa \& projeto de pesquisa: escolhendo entre cinco abordagens. Porto Alegre: Penso, 2014.

GASQUE, Kelley Cristine Gonçalves Dias. Letramento informacional: pesquisa, reflexão e aprendizagem. Brasília: FCI/UnB, 2012.

GLASSMAN, Jeremy; PROSCH, Marilyn; SHAO, Benjamin B. M. To monitor or not to monitor: effectiveness of a cyberloafing countermeasure. Information \& Management, Tempe, v. 52, n. 2, p. 170-182, 2015.

GOLEMAN, Daniel. Foco: a atenção e seu papel fundamental para o sucesso. Rio de janeiro: Objetiva, 2014.

GONÇALVES, Lucio L. Dependência digital: tecnologias transformando pessoas, relacionamentos e organizações. Rio de Janeiro: Barra Livros, 2017.

HAIGH, Thomas. The history of information technology. In: CRONIN, Blaise (ed.). Annual review of information science and technology, [s.l.], v. 45. New Jersey: Medford, 2011.

HJELSETH, Eilif. Exchange of relevant information in BIM objects defined by the role- and life-cycle information model. Architectural engineering and design management, [s.l.], v. 6, n. 4, p. 279-287, 2010. 
HOUAISS, Antonio; VILAR, M. de Salles. Dicionário Houaiss da língua portuguesa. Rio de Janeiro: Objetiva, 2009.

JAMALUDDIN, Hasmida.et al. Personal Internet use: the use of personal mobile devices at the workplace. Procedia - Social and Behavioral Sciences, [s.l.], v. 172, p. 495-502, 2015.

KHANSA, Lara. et al. Cyberloafing in the workplace: mitigation tactics and their impact on individuals' behavior. Information Technology and Management, Punjab, v. 19, n.4, p.197-215, 2017.

KNIGHT, Peter T. A internet no Brasil: origens, estratégia, desenvolvimento e governança. Bloomington: AuthorHouse, 2014.

LARA, Pablo Z. Manrique de. Fear in organizations: does intimidation by formal punishment mediate the relationship between interactional justice and workplace internet deviance? Journal of Managerial Psychology, Las Palmas, v. 21, n. 6, p. 580-592, 2006.

LAZARTE, Leonardo. Ecologia cognitiva na sociedade da informação. Ciência da Informação, Brasília, v. 29, n. 2, maio/ago, p. 43-51, 2000.

LIM, Vivien K. G.; CHEN, Don J. Q. Cyberloafing at the workplace: gain or drain on work? Behaviour \& Information Technology, Singapore, v. 31, n. 4, p. 343-353, 2012.

LIPKIN, Nicole; PERRYMORE, April. A geração Y no trabalho: como lidar com a força de trabalho que influenciará definitivamente a cultura da sua empresa. Rio de Janeiro: Elsevier, 2010.

McGARRY, Kevin. O contexto dinâmico da informação: uma análise introdutória. Brasília: Briquet de Lemos, 1999.

MILLION, Anthony J. et al. Opportunistic discovery of information and millennials: an exploratory survey. Proceedings of the American Society for Information Science and Tecnology, Quebec, v. 50, n. 1, p, 1-5, 2013.

MOURA JUNIOR, Pedro J.; HELAL, Diogo H. Profissionais e profissionalização em tecnologia da informação: indicativos de uma "metamorfose ambulante". In: ENCONTRO DE ADMINISTRAÇÃO DA INFORMAÇÃO, 4., 2013, Bento Gonçalves. Anais [...]. Bento Gonçalves, 2013.

PIJPERS, Guus. Information overload: a system for better managing everyday data. Nova Jersei: Wiley, 2010.

SANTOS, Boaventura de Sousa. Os tribunais e as novas tecnologias de comunicação e de informação. Sociologias, Porto Alegre, v. 7, n. 13, jan./jun., p,82-110, 2005. 
SARACEVIC, Tefko. The notion of relevance in information science: everybody knows what relevance is. But what is it really? New Carolina: Gary Marchionin, 2017.

SAVOLAINEN, Reijo. Elaborating the conceptual space of information-seeking phenomena. Information Research, Borås, v. 21, n. 3, set. 2016.

SAWITRI, Hunik S.R. Role of internet experience in moderating influence of work stressor on cyberloafing. Procedia - Social and Behavioral Sciences, [s.l.]v. 57, out., p. 320-324, 2012.

SCHAMBER, Linda; EISENBERG, Michael B; NILAN, Michael S. A reexamination of relevance: toward a dynamic, situational definition. Information Processing and Management, [s.l.], v. 26, n. 6, p. 755-776, 1990.

SHANTEAU, James. How much information does an expert use? Is it relevant? Acta Psychologica, Gante, v. 81, p. 75-86, 1992.

SPINK, Amanda; COLE, Charles. Human information behavior: integrating diverse foraging use. Journal of the American Society for Information Science and Technology, New York, v. 57, n. 1, jan., p. 25-35, 2006.

TARAPANOFF, Kira. Informação, conhecimento e inteligência em corporações: relações e complementaridade. In: TARAPANOFF, Kira (org.). Inteligência, informação e conhecimento. Brasília: IBICT, 2006.

TURKLE, Sherry. The flight from conversation. The New York Times, New York, 21 abr. 2012.

WILSON, Thomas, D. Information behaviour: an interdisciplinary perspective. Information Processing \& Management, [s.l.], v. 33, n. 4, p. 551-572, 1997.

WILSON, Thomas, D. Models in information behavior research. Journal of Documentation, London, v. 55, n.3, p. 249-270, 1999.

WILSON, Thomas, D. Human information behavior. Informing Science, [s.l.], v. 3, n. 2, p.49-55, 2000.

\title{
Impacts of irrelevant information seeking to the productivity of Information Technology professionals
}

\begin{abstract}
This article is the result of a doctoral research developed with professionals from the Information Technology infrastructure area, who work in the Distrito Federal (federal district). It uses a quantitative-qualitative methodology, with questionnaires application and individual interviews of semistructured nature. The results show that these professionals are connected most
\end{abstract}


of the time during the work and, in general, accessing information that is not relevant to their immediate task, a phenomenon called cyberloafing. The main reasons for the access are updates on the latest events, the reduction of stress level and the increase of the levels of technical knowledge. It has been recognized that cell phone access causes a distraction, yet many professionals have criticized their workplace for the practice of blocking websites and contents. The main impacts on the work are little use of the time for the development of the company's tasks and diversion of attention. Organizations need to adapt to this new reality and find management and control solutions rather than opting for the exclusive use of tools to block access to certain types of sites and applications.

Keywords: Information seeking. Cyberloafing. Irrelevant information. Relevant information. Information overload.

Recebido: $15 / 02 / 2018$

Aceito: 18/09/2018 